\section{P115 CIGARETTES SMOKE EXTRACT INDUCES INFLAMMATORY GENE EXPRESSION IN HUMAN BRONCHIAL EPITHELIAL CELLS}

M Alshehri, O Brand, A Alqarni, A Pasini, L Pang. Division of Respiratory Medicine, School of Medicine, University of Nottingham, (City Hospital), Nottingham, UK

\subsection{6/thoraxjnl-2017-210983.257}

Background Chronic obstructive pulmonary disease (COPD) is an inflammatory disorder of the respiratory tract characterised by airflow obstruction. It is increasingly recognised that the innate immune pattern-recognition receptors may contribute to airway inflammation in COPD in response to environmental factors such as cigarette smoke (CS). One pattern-recognition receptor that has recently come to attention in chronic airway disease is the cell surface receptor for advanced glycation end products (RAGE). RAGE also exists as a soluble form (sRAGE) that primarily functions as receptor decoy and an endogenous inhibitor of RAGE signalling. Clinical studies show that smokers with or without COPD have significantly greater levels of RAGE expression in airway epithelial cells compared with never smokers. However, the role of RAGE in mediating CSinduced inflammatory gene expression has not been understood. We hypothesise that CS can induce RAGE expression, sRAGE reduction, and inflammatory gene expression in human bronchial epithelial cells (BEAS-2B).

Method Confluent BEAS-2B cells were treated with different concentrations of Cigarette Smoke Extract (CSE) (1\%, 2.5\%, and 5\%) for 24 hours. Western blotting was used to assess protein expression of RAGE in cell lysate. ELISA was used to measure interleukin 6 (IL-6), CXCL1 (GRO- $\alpha$ ), CXCL5 (ENA78), CXCL8 (IL-8), CXCL10 (IP-10), CCL11 (eotaxin), and sRAGE in culture medium.

Result We found that IL-6 and CXCL8 releases were markedly increased by CSE in a concentration-dependant manner, but CXCL-1, CXCL5, CXCL10 and CCL11 could not be detected in both untreated and CSE-treated cells. Interestingly, RAGE was highly expressed in untreated cells and CSE treatment did not further increase its expression. Furthermore, sRAGE was also undetectable in both untreated and CSE-treated cells.

Conclusion These findings suggest that CSE can induce inflammatory gene expression in BEAS-2B cells. Further experiments are being conducted to explore the effect of CSE on other inflammatory gene expression and to investigate the role of RAGE in mediating CSE-mediated inflammatory response in BEAS-2B cells.

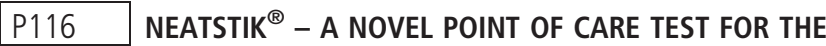 MEASUREMENT OF ACTIVE NEUTROPHIL ELASTASE IN PATIENTS WITH RESPIRATORY DISEASE}

${ }^{1} \mathrm{DF}$ McCafferty, ${ }^{1} \mathrm{KL}$ Moffitt, ${ }^{2} \mathrm{~W}$ Tong, ${ }^{1} \mathrm{TEG}$ Ferguson, ${ }^{1} \mathrm{C}$ Robb, ${ }^{3} \mathrm{SL}$ Martin, ${ }^{1} \mathrm{~B}$ Walker. ${ }^{1}$ ProAxsis Ltd, Belfast, UK; ${ }^{2} D C N$, Carlsbad, US; ${ }^{3}$ Queen's University, Belfast, UK

\subsection{6/thoraxjnl-2017-210983.258}

Introduction and Objectives Sputum levels of active neutrophil elastase (NE) are frequently elevated in respiratory diseases, such as bronchiectasis, chronic obstructive pulmonary disease (COPD), and cystic fibrosis (CF). Increased NE levels have been demonstrated to inversely correlate with pulmonary function, and risk of exacerbations. Active NE is also recognised as a biomarker of subclinical infection; however, no tools presently exist for its routine monitoring at home or in the clinic. Translation of the ProAxsis NE Activity Based Immunoassay (ABI) to a Point of Care (PoC) device, would facilitate routine monitoring of those patients at highest risk of upcoming exacerbations; enabling pre-emptive medical intervention, and mitigating the patient's risk of developing serious complications.

Methods Active NE levels in sputum samples $(n=10)$ were determined using the NE ABI (ProAxsis Ltd, Belfast), in accordance with manufacturer's instructions; followed by assessment using the NEATstik PoC test (threshold $10 \mu \mathrm{g} / \mathrm{ml}$ in sputum). For measurement of NE using NEATstik, sputum was diluted $\mathrm{x} 10$, gently rotated for $1 \mathrm{~min}$, an aliquot $(70 \mu \mathrm{l})$ was then transferred onto the test sample port of the device and allowed to develop for $10 \mathrm{~min}$, after which the signal intensity at the test-line was visually graded $(0-10)$.

Results NE ABI analysis of sputum revealed that 7 out of 10 samples under investigation contained active NE at levels above the NEATstik test threshold. All 7 samples were found to produce a strong positive test line on the PoC device. Moreover, no test line was visible for the remaining samples with active NE concentrations below $10 \mu \mathrm{g} / \mathrm{ml}$.

Conclusion Availability of NEATstik, the first highly sensitive and specific PoC test for the rapid detection of active NE in complex clinical samples, should enable the proactive management of multiple chronic respiratory diseases. It has the potential to assist in the identification of patients at highest risk of imminent exacerbations, and thus allow closer monitoring by their clinical team and/or pre-emptive treatment to avoid/minimise the impact of such exacerbations. Additionally, for those presenting with an ongoing exacerbation, the test facilitates patient stratification, with those most likely to respond to antibiotic therapy identified and their response to treatment assessed.

\section{P117 NEUTROPHIL CHEMOTAXIS IN THE SZ FORM OF ALPHA- 1 ANTITRYPSIN DEFICIENCY}

${ }^{1} S$ Vayalapra, ${ }^{2}$ AJA McGuinness, ${ }^{2}$ RA Stockley, ${ }^{2}$ AM Turner. ${ }^{1}$ College of Medical and Dental Sciences, University of Birmingham, Birmingham, UK; ${ }^{2}$ University Hospitals Birmingham, Birmingham, UK

\subsection{6/thoraxjnl-2017-210983.259}

Introduction Alpha-1 antitrypsin deficiency (AATD) is a genetic disorder which predisposes to the development of lung disease. The PiSZ phenotype is associated with a lower risk of disease and a pattern of emphysema more characteristic of patients with chronic obstructive pulmonary disease (COPD) than the PiZZ phenotype. Aberrant migration of neutrophils has been observed in stable COPD, possibly contributing to the pathogenesis. Neutrophils from PiZZ patients show a migratory phenotype similar to that of healthy controls and the migratory characteristics of PiSZ neutrophils have not been investigated.

Methods The chemotaxis of peripheral blood neutrophils in PiSZ patients was characterised using an Insall chamber and time-lapse video microscopy. Migratory characteristics of the neutrophils were compared with existing data from PiZZ and usual COPD patients. A marker of neutrophil elastase activity known as $\mathrm{A} \alpha-\mathrm{Val}^{360}$ was compared between patient groups and the relationship between patient characteristics and neutrophil migration was examined.

Results PiSZ neutrophils moved with a reduced velocity compared to cells from PiZZ patients in the presence of IL-8 